\title{
FBAUC: Favoriser la recherche clinique en urologie au Canada
}

\section{Robert Siemens, MD, FRCSC}

Rédacteur en chef adjoint du JAUC

Cite as: Can UrolAssoc J2011;5(5):303;001:10.5489/ cuaj.11213

\section{Références}

1. Khadaroo RG, Rotstein OD. Are clinician-scientists an endangered species? Barriers to clinician-scientist training. Clin Invest Med 2002;25:260-1.

2. Siemens DR, Punnen $S$, Wong J, et al. A survey on the attitudes towards research in medical school. BMC Med Educ 2010;22:10:4

3. Lander B, Hanley GE, Atkinson-Grosjean J. ClinicianScientists in Canada: Barriers to Career Entry and Progress. PLoS ONE 2010;5:e13168. doi:10.1371/ journal.pone.0013168
$\mathrm{L}$ es cliniciens-chercheurs jouent un rôle crucial dans le resserrement de l'écart entre la recherche biomédicale et la pratique clinique, formulant $d^{\prime}$ importantes questions de recherche axées sur les patients et traduisant ensuite les résultats d'études récentes en changements dans la pratique clinique. Au cours des 30 dernières années, de plus en plus d'articles et de discussions ont abordé la baisse du nombre de cliniciens-chercheurs ainsi que la tendance en recherche, pour ceux qui tentent encore de porter le flambeau, à s'éloigner de plus en plus des études basées sur les patients ${ }^{1}$. On peut percevoir une crainte grandissante voulant que la recherche s'enferme dans des projets exclusivement techniques, en particulier dans les spécialités chirurgicales, et se retrouve coupée du renouvellement constant permis par l'acquisition et la mise en application de nouvelles connaissances cliniques et en sciences fondamentales. Même si les raisons mises de l'avant pour expliquer la baisse du nombre de cliniciens-chercheurs sont multiples et variées, la plupart tournent autour de difficultés concernant la formation/l'encadrement, le temps et les aspects financiers. Bon nombre de ces obstacles perçus semblent se pointer tôt; en effet, plusieurs rapports publiés font état d'une attitude ambivalente envers la valeur de la recherche biomédicale dans les facultés de médecine et pendant la période de résidence².

Cela dit, de nombreuses initiatives au Canada commencent peut-être à corriger ce désintéressement pour la recherche manifesté par les médecins pratiquants. Certaines de ces initiatives incluent une participation précoce plus fréquente à des travaux de recherche pendant les études de médecine, le lancement de programmes de formation en recherche médicale, la promotion de la recherche par des bourses dans les sous-spécialités et la mise sur pied d'Instituts de recherche en santé du Canada (IRSC) et la création d'autres prix de recherche clinique sans but lucratif. Reflétant des rapports optimistes en provenance des États-Unis, Lander et ses collègues ont récemment rapporté les résultats d'une étude portant à croire que les cliniciens-chercheurs canadiens reçoivent davantage de subventions de recherche des IRSC et de bourses salariales ${ }^{3}$. Même si cette étude dresse un portrait plutôt optimiste, les obstacles auxquels sont confrontés nos nouveaux chirurgiens-chercheurs sont toujours considérables, avec, dans le meilleur des cas, des ressources universitaires ponctuelles pour corriger les problèmes de temps protégé afin de dégager le chercheur de ses responsabilités cliniques et de soutien salarial en l'absence de politiques ou programmes nationaux bien définis et bien coordonnés.

La Fondation des bourses de l'Association des urologues du Canada (FBAUC) a été mise sur pied en 1973 afin d'appuyer les chercheurs canadiens prometteurs en urologie et ainsi favoriser la productivité dans la recherche en urologie au Canada. Ce prix offert aux chercheurs en début de carrière joue un rôle crucial dans notre spécialité, permettant aux jeunes cliniciens-chercheurs d'obtenir un premier coup de pouce en termes de révision par des pairs et de soutien financier à un important tournant dans leur carrière. Au cours des 28 dernières années, la FBAUC a appuyé plus de 91 urologues universitaires et de nombreux médecins communautaires et étrangers, grâce à son engagement envers I'avancement de la recherche biomédicale en urologie. À la lecture du numéro du JAUC de ce mois-ci, on peut noter qu'au moins un ancien récipiendaire $d^{\prime}$ une bourse de la FBAUC est nommé à titre d'auteur pour chaque article. Avec un facteur d'impact de plus en plus élevé (1,72 à la dernière vérification), le JAUC est fier de contribuer à la mission de la communauté de recherche en urologie au Canada. En tant que rédacteur en chef adjoint du JAUC et président de la FBAUC, j'aimerais saluer et féliciter les récipiendaires de bourses de la FBAUC de cette année :

- Frédérick Pouliot - Université Laval

- Sero Andonian - Université McGill

- Rodney Breau - Université d'Ottawa

- Naji Touma - Université Queen's

- Blayne Welk - Université de l'Ouest de l'Ontario

- Jennifer Mickelson - Université de la Colombie-Britannique 